\title{
NIVEL DE CONOCIMIENTO RESPECTO A LA PLANIFICACIÓN FAMILIAR EN GESTANTES QUE ACUDIERON AL HOSPITAL UNIVERSITARIO SAN JOSÉ, POPAYÁN, COLOMBIA, 2014-2015
}

\section{Knowledge about contraception among pregnant women coming to San José Ulniversity Hospital in Popayán, Collombia, 2014-2015}

Edward Moreno-González, $M^{1}{ }^{1}$; Roberth Alirio Ortiz-Martínez, $M D, M S c^{2}$

Recibido: octubre 30/15 - Aceptado: septiembre 1/16

\section{RESUMEN}

Objetivos: determinar el nivel de conocimiento mínimo aceptable existente sobre planificación familiar que tienen las pacientes obstétricas que acuden al Hospital Universitario San José (HUSJ), en Popayán.

Materiales y métodos: estudio transversal en gestantes que acudieron a un hospital público universitario de referencia, de tercer nivel de complejidad, ubicado en el suroccidente de Colombia en los años 2014-2015. Se excluyeron aquellas con información inconsistente, las que por su situación de salud física o mental no pudieran contestar la encuesta y aquellas que no firmaron el consentimiento informado. Se definió como nivel de conocimiento mínimo aceptable aquellas encuestas con más de un $90 \%$ de ítems correctos. Tamaño de muestra: 361 pacientes. Se realizó muestreo aleatorio sistemático; se evaluaron las variables biológicas, sociales y demográficas. Respecto al conocimiento del método

\footnotetext{
Residente de tercer año, Universidad del Cauca, Popayán (Colombia). morenoedward@hotmail.com

2 Ginecoobstetra; magíster en Epidemiologia. Docente del Departamento de Ginecología y Obstetricia, Universidad del Cauca, Popayán (Colombia).
}

se indagó sobre aspectos relacionados con su uso, reacciones adversas y riesgo de falla. Se realizó análisis descriptivo.

Resultados: se evaluaron 361 mujeres, 94,46\% manifestaron conocer al menos un método de planificación. El 70,09\% de los embarazos no fueron planeados. Cerca de un cuarto de las pacientes estaban usando algún método de planificación familiar al momento del embarazo. Se encontró en un nivel de conocimiento mínimo del $90 \%$ en el 26,23\% de las pacientes, siendo el más bajo para anticonceptivos (11,85\%).

Conclusiones: a pesar de que el $94 \%$ de las gestantes entrevistadas conocían la existencia de métodos de planificación, en cuanto al conocimiento de cómo usarlos y las reacciones adversas asociadas, el $26 \%$ tuvo un nivel mayor al $90 \%$ en ambos aspectos.

Palabras clave: planificación familiar, nivel mínimo de conocimiento.

\section{ABSTRACT}

Objectives: To determine the level of basic acceptable knowledge on contraception among obstetric patients coming to the Hospital Universitario San José (HUSJ) in Popayán. 
Materials and methods: Cross-sectional study in pregnant women coming to a Level III referral public university hospital in Southwestern Colombia during 2014 and 2015. Patients with inconsistent information, or who could not complete the survey because of their mental or physical conditions, or those who did not sign inform consent, were excluded. Basic level of knowledge was defined as more than $90 \%$ correct items on the questionnaire. A systematic random sampling was performed, and the biological, social and demographic variables were analysed. In terms of knowledge of the contraception method, there were questions regarding use, adverse reactions and risk of failure. A descriptive analysis was performed.

Results: Of a total of 361 women included in the assessment, 94.46\% reported having knowledge of at least one contraception method. Of the pregnancies, $70.09 \%$ were unplanned. Close to one-fourth of the patients were using some form of contraception at the time of pregnancy. A basic level of knowledge of $90 \%$ was found in $26.23 \%$ of the patients, the lowest level being related to the knowledge about oral contraceptives $(11,85 \%)$. Conclusions: Despite the fact that $94 \%$ of the pregnant women interviewed knew of the existence of contraception methods, only $26 \%$ of them were found to have a level of knowledge greater than 90\% concerning their use and associated adverse reactions.

Keywords: Contraception, basic level of knowledge.

\section{INTRODUCCIÓN}

La planificación familiar (PF) es definida por la Organización Mundial de la Salud (OMS), como la posibilidad de un individuo o familia de determinar el tamaño y la estructura de su descendencia (1), implicando importantes beneficios para la salud reproductiva (2). Según la Encuesta Nacional de Salud (ENS) de 2010, Colombia es un país ejemplo en América Latina, ya que el conocimiento sobre planificación familiar es universal y está presente en todos los estratos socioeconómicos. Actualmente, la
ENS asegura que el $85 \%$ de las mujeres han usado al menos un método de planificación familiar durante su vida (3); por otro lado, se ha informado que en Colombia el $48 \%$ de las mujeres no saben que las empresas de aseguramiento en salud a las que están afiliadas (denominadas Entidades Promotoras de Salud - EPS) están obligadas a proporcionar los métodos anticonceptivos de manera gratuita; de la misma manera se evidencia que existen desigualdades en el acceso a métodos anticonceptivos de acuerdo con el nivel de riqueza: el $75 \%$ para las mujeres de mayor riqueza, frente al 67,5\% para las más pobres (4). Además, la información dada por las entidades oficiales (3) permite reconocer solo algunas características sobre el uso de métodos de planificación familiar, pero no profundiza, por ejemplo, en el nivel mínimo de conocimiento existente acerca de los diferentes métodos y las variables que determinan este nivel de conocimientos.

Este conocimiento podrá ser definido inicialmente como la información que se tiene sobre la existencia de los diferentes métodos de planificación familiar, pero debería incluir aspectos relacionados con el uso adecuado del método, sus reacciones adversas y los riesgos asociados a su uso $(1,5)$. Asimismo, es necesario conocer si la manera de usarlos es la adecuada, ya que de lo contrario implicaría malos resultados como el embarazo no deseado. Con respecto al nivel mínimo de conocimiento existente sobre los aspectos antes mencionados hay poca información, razón por la que se plantea el presente estudio, cuyo objetivo es hacer una aproximación al nivel de conocimiento mínimo aceptable existente que tienen las pacientes obstétricas en el país sobre planificación familiar.

\section{MATERIALES Y MÉTODOS}

Estudio transversal en gestantes que acudieron al servicio de obstetricia del Hospital Universitario San José (HUSJ) durante el periodo comprendido entre el 1 de marzo de 2014 al 1 de marzo de 2015, bien sea para atención de su parto o por algún otro motivo (aborto, amenaza de parto prematuro, entre 
otros). Esta es una institución pública que presta servicios de tercer nivel y es centro de referencia para población con aseguramiento subsidiado por el Estado, en el sistema de seguridad social en Colombia, en el departamento del Cauca, al suroccidente del país. Se excluyeron aquellas mujeres con información inconsistente, las que por su situación de salud física o mental no pudieran contestar la encuesta y las que no firmaron el consentimiento. Para el tamaño de muestra se aplicó la fórmula: $\mathrm{n}$ $=\mathrm{P} \times \mathrm{Q} /(\mathrm{E} / \mathrm{Z})^{2}$ y se tuvo en cuenta: población promedio de gestantes atendidas en los años previos (2100), porcentaje mínimo de conocimiento aceptable esperado del $50 \%$, un error tolerado del $5 \%$, corrección por población finita y por no respuesta del $20 \%$ y un nivel de confianza del $95 \%$, obteniendo así un tamaño de muestra de 361 pacientes. Se realizó muestreo aleatorio sistemático.

Procedimiento. Una vez que las participantes elegibles recibieron toda la información acerca del estudio y firmaron el consentimiento, fueron entrevistadas por personal entrenado que, además de aplicar listas de chequeo para cada método de planificación familiar, recolectó variables biológicas, sociales y demográficas, mediante un cuestionario semiestructurado, que fue diseñado por los investigadores, teniendo en cuenta literatura sobre el tema (6-13); este cuestionario fue revisado por expertos y ajustado por una prueba piloto en una población menor de similares características y en la misma institución; también se recolectó información de la historia clínica institucional. Las listas de chequeo por cada método de planificación familiar se basaron en los diferentes estudios mundiales realizados con un objetivo similar al presente estudio (14-18), y conceptos emitidos por la OMS en manuales dirigidos a proveedores de salud (5), usando el modelo de la escala Likert.

Se midieron variables como edad, escolaridad, raza, estrato socioeconómico, aseguramiento, religión, ocupación, procedencia, estado civil, nivel educativo, si el actual embarazo fue planeado o no, razón para no uso de métodos de planificación fa- miliar (en caso de no hacerlo). La edad se categorizó en menores de 19 años, de 20 a 34 (categoría de referencia) y mayores de 35; en cuanto a la raza, se consideró la raza blanca y mestiza en un solo grupo y se tomó como la categoría de referencia; de la misma manera, un nivel educativo mayor de 5 años. Respecto al conocimiento de un método específico de planificación, la lista de chequeo incluyó aspectos sobre asesoría médica previa, control rutinario que incluía control posquirúrgico cuando aplicaba, cuidados de conservación del medicamento, uso diario del medicamento, acciones en caso de olvido en los métodos hormonales y de barrera, conocimiento de efectos adversos y falla terapéutica, implicaciones sociales y culturales que impliquen barreras en el conocimiento. Con respecto a la variable objetivo del presente estudio se definió: nivel de conocimiento mínimo aceptable a la encuesta con más de un $90 \%$ de ítems correctos, según lista de chequeo correspondiente al método de planificación familiar que la paciente manifestó previamente conocer, sea por uso previo o por información adquirida. Se consideró el $90 \%$ como un conocimiento mínimo aceptable por consenso de expertos conformado por los docentes del Departamento de Ginecología y Obstetricia de la Universidad del Cauca.

Análisis estadístico. Una vez realizado el control de calidad de la información, se elaboró una base de datos en Excel que luego fue llevada al paquete estadístico Stata 9.0, donde se realizó el análisis final. Por último, para el porcentaje del nivel de conocimiento mínimo global aceptable existente se tomó como numerador el total de encuestas con un $90 \%$ de respuestas correctas y como denominador el total de encuestas contestadas (se debe tener en cuenta que una entrevistada puede responder que conoce más de un método, por tanto, el total de encuestas es mayor al total de pacientes); de la misma manera, para cada método de PF se realizó una encuesta y se calculó el número de pacientes con el nivel de conocimientos mínimo del 90\%, para ello se tuvo en cuenta como numerador el total de cuestionarios calificados como aceptables según el método evalua- 
do, y como denominador el total de gestantes que manifestaron conocer dicho método. Los datos se presentan mediante medidas de tendencia central y dispersión para variables continuas y proporciones para variables categóricas.

Aspectos éticos. El estudio recibió el aval del comité de ética del HUSJ de Popayán. Se respetó la confidencialidad de la información, y se solicitó consentimiento de la paciente para participar en el estudio.

\section{RESULTADOS}

El presente estudio se llevó a cabo entre el 1 de marzo 2014 al 1 de marzo del 2015, no se presentaron rechazos ni pérdidas, el análisis final se realizó con 361 pacientes. La edad promedio de la población bajo estudio fue de 26,5 años ( $\mathrm{DE} \pm 6,68$ ), la mayor parte proveniente de área rural, con nivel educativo menor a 5 años, se dedican al cuidado del hogar y pertenecientes al régimen subsidiado por el Estado, con unión estable y de raza mestiza (tabla 1). Con respecto a si conocen o no los métodos de planificación familiar, el 94,46\% manifestaron conocer al menos un método. Los métodos que la población estudiada manifestó conocer más fueron los inyectables mensuales, seguidos por los trimestrales y los anticonceptivos orales, y con menor frecuencia los de emergencia (tabla 2). El 70,09\% de los embarazos no fueron planeados. Cerca de un cuarto de las pacientes estaba usando algún método de planificación familiar al momento del embarazo. Un total de 172 pacientes manifestaron no usar métodos de planificación familiar antes del embarazo, las razones más frecuentes para su no uso fueron: no considerarlos necesarios $(38,95 \%)$ y temor a los efectos adversos (36,63\%) (tabla 2).

Respecto al nivel de conocimientos mínimo, 437 de 1229 encuestas (26,23\%) tuvieron el nivel de conocimiento global igual o superior al $90 \%$. Al evaluar el porcentaje del nivel mínimo de conocimiento por método de planificación familiar se encontró que el menor porcentaje corresponde a los anticonceptivos orales y el mayor correspondió a la ligadura de trompas (tabla 3). En cuanto al conocimiento de cómo usarlos y las reacciones adversas asociadas, el $26 \%$ tuvo un nivel mayor al $90 \%$ en ambos aspectos.

\section{DISCUSIÓN}

El presente estudio mostró que el 94,46\% conocía al menos la existencia de un método de PF; sin embargo, respecto al nivel de conocimiento igual o superior al $90 \%$ se observó que el 26\% tienen este nivel y en anticoncepción hormonal el nivel es solo del $11 \%$.

Nuestros hallazgos sobre el conocimiento de la existencia de los métodos de planificación son similares a los obtenidos en la ENS del 2010 (3), donde se encontró un 93,5\% de conocimiento sobre algún método. Al individualizarlos se observó que el conocimiento es mucho menor en el presente estudio que los reportados por la ENS.

El nivel de conocimiento mínimo adecuado sobre planificación familiar ha sido un área poco explorada en nuestro medio, pues la mayoría de estudios investigan el uso actual o el conocimiento sobre la existencia de métodos de planificación familiar, pero es escaza la información respecto a si las usuarias los usan en forma adecuada: lo mismo ocurre para el nivel de conocimiento mínimo adecuado. Actualmente, la mayoría de estudios internacionales aborda el tema desde una perspectiva del posible uso a futuro de algún método anticonceptivo y la percepción subjetiva del mismo (14-18), pero no aporta información adicional importante en el campo del conocimiento adecuado en su uso. En un estudio publicado en 2015, realizado en mujeres jóvenes en Estados Unidos mediante el formato de encuesta, se evidenció un conocimiento bajo y medio en el 70,4\% de las pacientes (14), hallazgo similar al encontrado en el presente estudio, pues en el 73,7\% de los casos el conocimiento es inadecuado. Por otro lado, en España se realizó un estudio transversal en población menor de 20 años y de ambos sexos, encontrándose un porcentaje de conocimiento real del 17,80\% (19), 


\section{Tabla 1.}

Características sociodemográficas de las pacientes gestantes encuestadas para evaluación del nivel mínimo de conocimiento del uso de métodos de PF del HUSJ, Popayán, Colombia, 2014-2015

\begin{tabular}{|c|c|c|}
\hline Variable & Frecuencia & Porcentaje \\
\hline \multicolumn{3}{|l|}{ ESE } \\
\hline Medio & 53 & 14.68 \\
\hline Bajo & 308 & 85,32 \\
\hline \multicolumn{3}{|l|}{ Zona de vivienda } \\
\hline Urbana & 204 & 56.51 \\
\hline Rural & 157 & 43,49 \\
\hline \multicolumn{3}{|l|}{ Nivel educativo } \\
\hline$>5$ años & 283 & 78.39 \\
\hline$\leq 5$ años & 78 & 21,61 \\
\hline \multicolumn{3}{|l|}{ Estado civil } \\
\hline Estable & 288 & 79.78 \\
\hline No estable & 73 & 20,22 \\
\hline \multicolumn{3}{|l|}{ Ocupación } \\
\hline Ama de casa & 187 & 51.8 \\
\hline Empleada & 66 & 18,28 \\
\hline Agricultora & 15 & 4,16 \\
\hline Independiente & 30 & 8,31 \\
\hline Estudiantee & 63 & 17,45 \\
\hline \multicolumn{3}{|l|}{ Etnia } \\
\hline Blanco - mestizo & 265 & 73.41 \\
\hline Negra - indígena & 96 & 26,59 \\
\hline \multicolumn{3}{|l|}{ Seguridad social } \\
\hline Contributivo & 102 & 28.25 \\
\hline Subsidiado & 259 & 71,75 \\
\hline \multicolumn{3}{|l|}{ Religión } \\
\hline Católica & 298 & 82.55 \\
\hline Otras & 57 & 15,79 \\
\hline Ninguna & 6 & 1,66 \\
\hline
\end{tabular}

Fuente: estudio de los autores.

PF: planificación familiar

HUSJ: Hospital Universitario San José 
Tabla 2.

Caracterización sobre el conocimiento de la existencia y el uso de métodos de planificación de las pacientes gestantes encuestadas en el HUSJ, Popayán, Colombia, 2014-2015

\begin{tabular}{|c|c|c|}
\hline Variable & Frecuencia & Porcentaje \\
\hline \multicolumn{3}{|l|}{ Conoce algún método de PF } \\
\hline Sí & 341 & 94,46 \\
\hline No & 20 & 5.54 \\
\hline \multicolumn{3}{|l|}{ Embarazo planeado } \\
\hline Sí & 108 & 29,91 \\
\hline No & 253 & 70.09 \\
\hline \multicolumn{3}{|l|}{ Uso de PF antes del embarazo } \\
\hline Sí & 81 & 22.44 \\
\hline No & 172 & 47,65 \\
\hline \multicolumn{3}{|l|}{ Razón para no uso de PF $(n=172)$} \\
\hline Desconocimiento & 20 & 11,63 \\
\hline Mi religión no lo permite & 2 & 1,16 \\
\hline Efectos adversos & 63 & 36,63 \\
\hline Mi médico tratante los suspendió & 14 & 8,14 \\
\hline Mi pareja los prohíbe & 2 & 1,16 \\
\hline No dispongo de recursos económicos & 4 & 2,33 \\
\hline No los considero necesarios & 67 & 38,95 \\
\hline \multicolumn{3}{|l|}{ Conoce los anticonceptivos orales } \\
\hline Sí & 253 & 70.08 \\
\hline No & 108 & 29,92 \\
\hline \multicolumn{3}{|l|}{ Conoce la inyección mensual } \\
\hline Síi & 265 & 73.41 \\
\hline No & 96 & 26,59 \\
\hline \multicolumn{3}{|l|}{ Conoce la inyección trimestral } \\
\hline Sí & 257 & 71.19 \\
\hline No & 104 & 28,81 \\
\hline \multicolumn{3}{|l|}{ Conoce el implante subdérmico } \\
\hline Sí & 217 & 60.11 \\
\hline No & 144 & 39,89 \\
\hline \multicolumn{3}{|l|}{ Conoce el DIU } \\
\hline Sí & 236 & 65.37 \\
\hline No & 125 & 34,63 \\
\hline \multicolumn{3}{|l|}{ Conoce el condón } \\
\hline Sí & 223 & 61.77 \\
\hline No & 138 & 38,23 \\
\hline \multicolumn{3}{|l|}{ Conoce la ligadura de trompas } \\
\hline Sí & 136 & 37.67 \\
\hline No & 225 & 62,33 \\
\hline \multicolumn{3}{|l|}{ Conoce los métodos de emergencia } \\
\hline Sí & 79 & 21.88 \\
\hline No & 282 & 78,12 \\
\hline
\end{tabular}

Fuente: estudio de los autores.

Hospital Universitario San José 


\begin{tabular}{|c|c|c|}
\hline \multirow[b]{2}{*}{ Método de planificación" } & \multicolumn{2}{|c|}{ Adecuado*: } \\
\hline & n & $\%$ \\
\hline Anticonceptivos orales & 30 & 11,85 \\
\hline Inyección mensual & 70 & 26,42 \\
\hline Inyección trimestral & 60 & 23,35 \\
\hline Implante subdérmico & 49 & 22,58 \\
\hline DIU & 59 & 25,00 \\
\hline Condón & 82 & 36,77 \\
\hline Ligadura de trompas & 62 & 45,59 \\
\hline Métodos de emergencia & 25 & 31,65 \\
\hline Total de conocimiento & 437 & 26,23 \\
\hline
\end{tabular}

Fuente: estudio de los autores.

Hospital Universitario San José

* Solo se incluyen las pacientes que manifestaron conocer dicho método, una paciente puede manifestar conocer más de un método..

** Total de cuestionarios que cumplían al menos un $90 \%$ de la lista de chequeo de dicho método.

menor al del presente estudio. Nuevos hallazgos sugieren que la principal barrera para el uso de los métodos de planificación familiar es el desconocimiento sobre su uso, tanto a nivel individual como comunitario (15). Este desconocimiento se asocia principalmente a un bajo nivel educativo (14) y al uso previo de métodos de planificación reversibles y de larga duración (16); este hallazgo es similar al encontrado en esta investigación, sobre todo en lo que respecta al nivel educativo. Un estudio evaluó la correlación entre el conocimiento y la aceptabilidad del método, y concluyó que deben desarrollarse estrategias educativas que permitan mejorar el uso de los métodos de planificación (17).

Esta investigación tiene como fortalezas el tamaño de la muestra, la no existencia de pérdidas, la realización de una prueba piloto y entrenamiento a los encuestadores, disminuyendo así el sesgo de información. Como debilidades, un posible sesgo de selección debido a que la población pertenece solo al tercer nivel de atención y solo se incluyó población gestante. Además, se requiere validar el cuestionario desde el punto de vista de la validez de contenido.

\section{CONCLUSIÓN}

El nivel de conocimiento mínimo, tanto global como individual, de los métodos de planificación familiar es bajo en la población estudiada; por esta razón, son necesarios más estudios con metodología diferente que permitan inferir causalidad sobre el porqué de este bajo conocimiento, y de esta manera tomar medidas acerca de este tópico.

\section{REFERENCIAS}

1. Organización Mundial de la Salud. Planificación familiar. OMS; 2011 [visitado 2016 Jun 10]. Disponible en: http://www.who.int/mediacentre/factsheets/ fs $351 /$ es/index.html.

2. Palma Y, Rivera G. La planificación familiar en México. En: Langer A, Tolbert K, editores. Mujer: sexualidad y salud en México. México D.F.: The population council y EDAMEX S.A; 1996. p. 153-69. 
3. Profamilia. Salud Sexual y Reproductiva en Colombia. Encuesta Nacional de Demografía y Salud; 2010.

4. Organización de las Naciones Unidas. 222 millones de mujeres no tienen acceso a la planificación familiar en el mundo. ONU; 2012 [visitado 2016 Jun 10]. Disponible en: http://nacionesunidas org co/blog/2012/11/14/222-millones de-mujeres-notienen-acceso-a-la-planificacion-familiar-en-elmundo/

5. Organización Mundial de la Salud. Planificación familiar. Un manual mundial para proveedores; 2011 [visitado 2016 Jun 10]. Disponible en: http://www. who.int/reproductivehealth/publications/family_ planning/9780978856304/es/

6. Turok D. A la búsqueda de una mejor contracepción: métodos futuros. Clínicas Obstétricas y Ginecológicas de Norte América. 2007;34:137-66.

7. Organización Mundial de la Salud. Criterios médicos de elegibilidad para el uso de anticonceptivos. 3a ed. Ginebra: OMS; 2005.

8. Kulier R, Boulvain M, Walker D, De Candolle G, Campana A. Minilaparotomy and endoscopic techniques for tubal sterilization. Cochrane Database of Syst Rev. 2004;(3):CD001328.

9. Diaz S. Contraceptive technology and family planning services. Int J Gynaecol Obstet. 1998;63(Suppl 1):S85-90.

10. Kavanaugh ML, Jerman J, Ethier K, Moskosky S. Meeting the contraceptive needs of teens and young adults: youth-friendly and long-acting reversible contraceptive services in U.S. family planning facilities. J Adolesc Health. 2013;52:284-92. doi: 10.1016/j. jadohealth.2012.10.276.

11. Ramírez A, Avecilla A. Novedades en anticoncepción. FMC. Form Med Contin Aten Prim. 2004;11:314-31.

12. Grimes DA, Lopez LM, Schulz FK. Antibiotic prophylaxis for intrauterine contraceptive device insertion. Cochrane Database of Syst Rev. 1999; (3):CD001327.

13. Black A, Francoeur D, Rowe T, Collins J, Miller D, Brown T, et al. SOGC clinical practice guidelines: Canadian contraception consensus. J Obstet Gynaecol Can. 2004;26:219-96.

14. Gomez AM, Hartofelis EC, Finlayson S, Clark JB. Do Knowledge and Attitudes Regarding Intrauterine Devices Predict Interest in Their Use? Womens Health Issues. 2015;25:359-65. doi: 0.1016/j.whi. 2015. 03.011.

15. Hall KS, Ela E, Zochowski MK, Caldwell A, Moniz M, McAndrew L, et al. "I don't know enough to feel comfortable using them:" Women's knowledge of and perceived barriers to long-acting reversible contraceptives on a college campus. Contraception. 2016;93:556-64. doi: 10.1016/j.contraception.2016.02.007.

16. Gosavi A, Ma Y, Wong H, Singh K. Knowledge and factors determining choice of contraception among Singaporean women. Singapore Med J. 2016 Jan 4. doi: 10.11622/smedj.2015181 [Epub ahead of print].

17. Hoopes AJ, Ahrens KR, Gilmore K, Cady J, Haaland WL, Amies Oelschlager AM, et al. Knowledge and Acceptability of Long-Acting Reversible Contraception Among Adolescent Women Receiving School-Based Primary Care Services. J Prim Care Community Health. 2016;7:165-70. doi: 10.1177/2150131916641095.

18. Kathpalia SK. Emergency contraception: Knowledge and practice among women and the spouses seeking termination of pregnancy. Med J Armed Forces India. 2016;72:116-9. doi: 10.1016/j.mjafi.2015.09.012.

19. Santín C, Torrico E, López MJ, Revilla C. Conocimiento y utilización de los métodos anticonceptivos y su relación con la prevención de enfermedades de transmisión sexual en jóvenes. Anales de Psicología. 2003;19:81-90. 